\title{
Sex-Dependent Differences in Symptom-Related Disability Due to Lumbar Spinal Stenosis
}

This article was published in the following Dove Press journal:

Journal of Pain Research

\section{Raffael Peteler $\mathbb{D}^{1,2}$ \\ Paul Schmitz ${ }^{2}$ \\ Martin Loher ${ }^{2}$ \\ Petra Jansen ${ }^{3}$ \\ Joachim Grifka' \\ Achim Benditz (D)}

'Department of Orthopedics, University Medical Center Regensburg, Asklepios Klinikum Bad Abbach, Bad Abbach, Bavaria, Germany; ${ }^{2}$ Department of Trauma Surgery, Caritas-Krankenhaus St. Josef, Regensburg, Bavaria, Germany; ${ }^{3}$ Department of Sport Science, University of Regensburg, Regensburg, Bavaria, Germany
Correspondence: Achim Benditz Kaiser-Karl-V.-Allee 3, Bad Abbach, 93077, Germany

Email achim.benditz@ukr.de
Study Design: Retrospective observational study.

Objective: The objective of this study is to identify possible sex-dependent differences in symptom-related disability in patients with lumbar spinal stenosis.

Methods: 103 consecutive outpatients (42 men and 61 women) with lumbar spinal stenosis were assessed on the basis of their medical history, the physical examination, and a series of questionnaires including the Oswestry Disability Index (ODI), the Roland Morris Disability Questionnaire (RMDQ), the Patient Health Questionnaire module 9 (PHQ-9), and the Depression Anxiety Stress Scales (DASS). Narrowing of the spinal canal was graded according to the method established by Schizas. Parameters were statistically analyzed according to the biological sex of the patients. The influence of the variables on the disability scores was analyzed by means of a multivariate regression model.

Results: Symptom severity was equally distributed between men and women. Female patients showed higher RMDQ and ODI scores as well as significantly higher intermediate depression scores. The confounding variables age, pain chronicity, and psychological affection as well as the symptoms level of pain and paresis were dependent on patient sex.

Conclusion: The study shows sex-depended differences in the perception of symptoms of lumbar spinal stenosis and disability of life. The findings suggest that the main mediators are pain perception and psychological influences on the quality of life.

Keywords: LSS, quality of life, disability, sex differences, pain

\section{Introduction}

Lumbar spinal stenosis (LSS) is a complex disease with a wide variety of symptoms and anatomic pathologies. Besides pain in the lower back and buttocks, the main symptom is neurogenic intermittent claudication. The combination of degenerative changes such as bulging of the discs, facet joints with osteophytes, and hypertrophy of the ligamentum flavum (LF) leads to the various known clinical appearances of LSS. ${ }^{1,2}$ The subjective perception of symptoms often does not correlate with the objective expression. ${ }^{3}$ Therefore, therapeutic decision-making is based on multiple factors including symptom severity, disability in daily life, and radiological criteria. $^{2}$

Male and female patients are known to differ in symptom-related disability as women have shown higher pain perception and elevated depression scores. ${ }^{3}$ Because pain may lead to depression, particularly in chronic conditions, ${ }^{4,5}$ it is important to identify symptom-related disability to initiate individualized therapies. Symptom-related disability poses a major challenge to health systems, not only from a medical but also from a socio-economic point of view. ${ }^{6,7}$ Therefore, the aim 
of this study was to examine sex-dependent differences in symptom-related disability of patients with LSS and to identify the factors influencing disability.

\section{Patients and Methods}

\section{Study Design}

This observational study consisted of a retrospective data analysis that was based on prospective data obtained at a single point in time from self-administered and selfreported health questionnaires (SSHQ) as well as from physical and radiological assessment.

\section{Patients and Data Collection}

For ten consecutive months, all outpatients with diagnosed LSS who had presented at an orthopedic clinic of a German university hospital were assessed within an LSS study program. The data of the current study were obtained from this data pool. A total of 103 patients were included in this study, the inclusion criteria were clinical and radiological diagnosed LSS. Exclusion criteria were cervical or thoracic spinal stenosis, vascular diseases, disc prolapse, diseases affecting the central or peripheral nerve system including previous spinal surgery, and preexisting or pre-known psychiatric disorders. Two patients were excluded post hoc because of missing radiological images. The analyzed cohort contained $\mathrm{N}=42$ (40.7\%) male patients and $\mathrm{N}=61$ (59.3\%) female patients. The demographics of the study cohort are shown in Table 1.
The study was approved by the Ethics Committee of the University of Regensburg, Germany (14-101-0136) and carried out in accordance with the approved guidelines of the Helsinki Declaration of 1975. Written informed consent was obtained from all study subjects.

\section{Clinical Measures}

All SSHQ screening tools are validated in the German version. Psychological aspects were analyzed with the PHQ- $9^{8}$ and the DASS module. ${ }^{9}$ The PHQ-9 is a 9-item depression module of the Patient Health Questionnaire. Each answer is graded from 0 to 4 and then summed up. The scale spans from 0 to 36, and the cut-off for depressive disorder is 4 . The DASS module comprises seven items graded from 0 to 3 with a combined maximum score of 21 . The range is subdivided into five categories from "normal" to "extreme severe", and the cut-off for light depressive disorder is 4 .

Disability was evaluated by means of the Oswestry Disability Index $(\mathrm{ODI})^{10}$ and the Roland Morris Disability Questionnaire (RMDQ). ${ }^{11}$ The ODI consists of ten questions on back pain-related impairment in daily life graded from 0 to 5 and then summed up. Disability may then be objectified using percental impairment respective to the maximum of 50 points. The range is subdivided into five equal-ranging segments from "minimal impairment" to "bedridden". The RMDQ consists of 24 items describing impairment due to back pain. To evaluate the level of disability, all positively answered items are summed up. No cut-off values or subdivisions are applied to this scale.

Table I Comparison of Baseline Data, Symptom Severity, Depression Scores, and Disability Scores of the Patients (Range in Brackets)

\begin{tabular}{|c|c|c|c|}
\hline & Men & Women & $\mathbf{p}$ \\
\hline $\mathrm{N}$ & 42 & 61 & \\
\hline Age (years) & $66(41-80)$ & $72(50-90)$ & 0.021 \\
\hline BMI & $30(2 I-42)$ & $30(16-45)$ & 0.558 \\
\hline Comorbidities (0: I: 2: 3) (\%) & 17: $57: 21: 5$ & 15: 60: 17: 8 & 0.738 \\
\hline Grade of spinal stenosis according to Schizas (A: B: C: D) (\%) & 10: $33: 45: 12$ & II.: $31: 46: 12$ & 0.969 \\
\hline Duration of symptoms (I: 2: 3: 4) (\%) & 7: 12: $21: 60$ & 5: 8: $26: 61$ & 0.580 \\
\hline Pain chronicity (MPSS grade I: $2: 3$ ) (\%) & 36: $52: 12$ & 16: $56: 28$ & 0.023 \\
\hline DASS & $3(0-14)$ & $5(0-15)$ & 0.067 \\
\hline PHQ-9 & $5(0-13)$ & $8(0-19)$ & 0.005 \\
\hline Level of pain (NRS) & $8(1-10)$ & $8(0-10)$ & 0.779 \\
\hline Pain irradiation (none: only back pain: radicular leg pain: pseudoradicular leg pain) (\%) & 0: 17: $40: 43$ & 2: 5: $54: 39$ & 0.649 \\
\hline Claudication (none: light: moderate: severe) (\%) & 17: 7: 29: 47 & |3: 7: 29: 51 & $0.88 \mathrm{I}$ \\
\hline Hyposensation (none: radicular: irregular) (\%) & 59: 17: 24 & 47: 23: 30 & 0.307 \\
\hline Paresis according to Janda $(5 / 5: 4 / 5:>4 / 5)(\%)$ & 69: $12: 19$ & 80: 20: 0 & 0.445 \\
\hline ODI & $41(10-82)$ & $47(7-80)$ & 0.109 \\
\hline RMDQ & $14(0-23)$ & $16(6-22)$ & 0.063 \\
\hline
\end{tabular}


The comorbidities were categorized into groups: Tumor-associated, Pulmonary, Cardial, Abdominal, Renal, Endocrinological, Dermal and Musculoskeletal Comorbidities. The influence of comorbidities on the quality of life was self-reported by patients and graded between 0 for no reduction of quality of life and 3 for high reduction of quality of life. For analysis, the mean of all categories was used.

The duration of symptoms was divided into four categories: 1 for a duration less than 6 weeks, 2 for a duration between 6 weeks and 6 months, 3 for a duration between 6 months and 2 years and 4 for a duration over 2 years.

Chronicity of pain was evaluated using the Mainz Pain Staging System (MPSS) ${ }^{12}$ that consists of four axes (Time, Location, Medication, and Patient career) and is graded in three levels. 1 is given for low chronicity, 2 for intermediate, and 3 for high chronicity.

Muscle strength was graded according to the method established by Janda. ${ }^{13}$ The segment-indicating muscles of segments L3 - S1 were assessed. The scale is subdivided into 6 grades ranging from $5 / 5$ to $0 / 5$. $5 / 5$ equals full muscle strength, whereas each grade below $5 / 5$ equals loss of muscle function by $25 \%$ measured by resistance and gravity. 1/5 equals muscle fibrillation and $0 / 5$ no muscle reaction at all.

The Numeric Rating Scale (NRS) is a 0-to-10 scale depicting a patient's level of pain in which 0 equals no pain and 10 the highest pain imaginable. Both average leg pain and back pain over the last months prior to elicitation were assessed separately. In case of difference within the two values, the higher value was used for statistical analysis.

The severity of claudication symptoms was measured by the walking time until the onset of symptoms. The walking time was self-reported by the patients. "Severe" represents up to 5 minutes, "moderate" up to 20 minutes, and "light" over 20 minutes.

The pain irradiation was elicitated by self-reported information by the patients and validated through physical examination by one experienced spine surgeon. If the irradiation could not be localized to explicit segments, it was rated pseudoradicular. The same technique was applied to the elicitation of hyposensation.

\section{Radiological Imaging}

Narrowing of the spinal canal was assessed by means of T2-weighted MRI scans of the spine. The radiological severity of LSS was graded according to the classification by Schizas ${ }^{14}$ that grades the ratio of cerebrospinal fluid (CSF) to the nerve roots and the spinal canal in axial imaging. At grade A1-A4, the nerve roots are fully enclosed by CSF, and grade B shows a reduced amount of CSF without entrapping the nerve roots. At grade C, the nerve roots are compressed without any visible CSF, but epidural fat can be found. At grade D, neither nerval tissue nor epidural fat are detectable due to extensive narrowing of the spinal canal. In the case of multi-level spinal stenosis, the level with the highest grade was considered for analysis.

\section{Statistical Analysis}

Statistical analysis was done with SPSS Statistics 23 (IBM Corp.). The independent $t$-test was used for analyzing variable distribution, and the Levene test for analyzing variance of non-linear variables. Results are shown in Table 1. The influence of variables on the variance was evaluated using the ANCOVA test. For correlation analysis, the two-sided correlation method by Pearson was used. All correlations with a p-value of $\leq .05$ are considered significant. The effect size was graded according to Cohen. ${ }^{15}$

The initial correlations of LSS symptoms with the disability scores of ODI and RMDQ according to patient sex were secondly controlled by adjusting to the cofactor variables (see Table 2). To analyze the influence of the different variables on the disability scores, a hierarchical multivariate linear regression model was used in respect to patient sex (see Table 3). Three blocks were subsequently added to this model. The first block consists of the baseline variables (age, BMI, radiological grading, pain chronicity), the second comprises the psychological screening scores (DASS, PHQ-9), and the third the somatic LSS symptoms (level of pain, pain irradiation, claudication, hyposensation, paresis) (see Table 4).

\section{Results}

\section{First Block: Baseline Data}

The female study group showed a moderately higher level of disability in both the ODI and RMDQ than the male study group, although it does not differ on a significant level (see Table 1). After adjustment for the baseline variables age and pain chronicity, there was no significant difference in ODI $(p=0.657)$ and RMDQ $(p=0.322)$ between the study groups. The comorbidities had no significant influence on the quality of life. 
Table 2 Variable Correlation with the Disability Scores ODI and RMDQ According to Patient Sex

\begin{tabular}{|c|c|c|c|c|c|c|c|c|}
\hline & \multicolumn{4}{|c|}{ RMDQ } & \multicolumn{4}{|c|}{ ODI } \\
\hline & \multicolumn{2}{|c|}{ Men } & \multicolumn{2}{|c|}{ Women } & \multicolumn{2}{|c|}{ Men } & \multicolumn{2}{|c|}{ Women } \\
\hline & $\mathbf{r}$ & $\mathbf{p}$ & $\mathbf{r}$ & $\mathbf{p}$ & $\mathbf{r}$ & $\mathbf{p}$ & $\mathbf{r}$ & $\mathbf{p}$ \\
\hline Age & 0.307 & 0.048 & 0.053 & 0.685 & 0.319 & 0.039 & 0.030 & 0.820 \\
\hline BMI & 0.104 & 0.521 & 0.117 & 0.378 & -0.152 & 0.350 & 0.158 & 0.233 \\
\hline Comorbidities & 0.076 & 0.241 & 0.158 & 0.083 & 0.122 & 0.204 & 0.251 & 0.103 \\
\hline Grade of spinal stenosis & 0.242 & 0.122 & 0.177 & 0.172 & 0.135 & 0.395 & 0.162 & 0.212 \\
\hline Duration of symptoms & -0.049 & 0.759 & 0.311 & 0.016 & -0.067 & 0.674 & 0.314 & 0.014 \\
\hline Pain chronicity & 0.265 & 0.089 & 0.407 & 0.001 & 0.336 & 0.029 & 0.537 & 0.000 \\
\hline DASS & 0.477 & 0.000 & 0.497 & 0.000 & 0.304 & 0.050 & 0.524 & 0.000 \\
\hline PHQ-9 & 0.344 & 0.026 & 0.551 & 0.000 & 0.202 & 0.199 & 0.531 & 0.000 \\
\hline Level of pain $(*)$ & $0.242(0.507)$ & $0.123(0.006)$ & $0.525(0.302)$ & $0.000(0.049)$ & $0.219(0.423)$ & $0.163(0.025)$ & $0.447(0.315)$ & $0.000(0.040)$ \\
\hline Pain irradiation $(*)$ & $0.015(-0.011)$ & $0.924(0.95 \mathrm{I})$ & $-0.266(-0.191)$ & $0.039(0.170)$ & $-0.020(0.023)$ & $0.898(0.895)$ & $-0.265(-0.167)$ & $0.039(0.23 \mathrm{I})$ \\
\hline Claudication $(*)$ & $0.236(0.153)$ & $0.132(0.439)$ & $0.032(-0.191)$ & $0.806(0.219)$ & $0.069(0.128)$ & $0.655(0.515)$ & $-0.012(-0.150)$ & $0.926(0.335)$ \\
\hline Hyposensation $(*)$ & $0.067(-0.128)$ & $0.674(0.517)$ & $0.258(0.238)$ & $0.045(0.124)$ & $-0.127(-0.234)$ & $0.423(0.230)$ & $0.298(0.316)$ & $0.020(0.039)$ \\
\hline Paresis $(*)$ & $0.073(0.082)$ & $0.646(0.667)$ & $0.359(0.275)$ & $0.004(0.074)$ & $0.204(0.112)$ & $0.194(0.570)$ & $0.456(0.329)$ & $0.000(0.031)$ \\
\hline
\end{tabular}

Note: *Adjusted for age, BMI, grade of spinal stenosis, pain chronicity, and DASS and PHQ-9 scores.

Table 3 Hierarchical Multivariate Regression Analysis According to Patient Sex

\begin{tabular}{|l|c|c|c|c|c|c|c|c|}
\hline & \multicolumn{4}{|c|}{ RMDQ } & \multicolumn{4}{c|}{ ODI } \\
\cline { 2 - 9 } & \multicolumn{2}{|c|}{ Men } & \multicolumn{2}{c|}{ Women } & \multicolumn{2}{c|}{ Men } & \multicolumn{2}{c|}{ Women } \\
\cline { 2 - 9 } & CR2 & P & cR2 & P & cR2 & P & cR2 & P \\
\hline Block 1 & 0.083 & 0.134 & 0.172 & 0.006 & 0.067 & 0.172 & 0.330 & 0.000 \\
Block 2 & 0.244 & 0.016 & 0.424 & 0.000 & 0.090 & 0.167 & 0.546 & 0.000 \\
Block 3 & 0.295 & 0.026 & 0.503 & 0.000 & 0.109 & 0.214 & 0.613 & 0.000 \\
\hline
\end{tabular}

The average age in the male study group was significantly lower than that in the female study group. Patient age only showed a significant correlation with the ODI and RMDQ scores in the male study group, indicating a congruent increase in disability with age, whereas the female study group showed a disproportional equal distribution of disability (see Figure 1).

Distribution of the BMI and the radiological grading of LSS did not significantly differ between the two study groups. Independent of patient sex, the BMI and the radiological grading were not significantly correlated with the ODI and RMDQ scores.

The duration of symptoms did not differ significantly between male and female patients. However, pain chronicity differed significantly between the two study groups as chronicity scores were higher for women. Pain chronicity had a very effective and highly significant correlation with the disability scores in the female study group and a low correlation in the male study group. Multivariate regression analysis only showed a significant influence of the baseline data on disability in the female study group. Out of the variables within the block, solely pain chronicity had a significant influence on disability in the female study group.

\section{Second Block: Psychological Scores}

The psychological scores showed significantly higher partial affection in the female study group. On average, the DASS score only showed slight depressive disorder in the female study group, whereas the PHQ-9 score indicated an average depressive affection in both study groups (see Table 1).

The correlation of DASS and PHQ-9 with the disability scores showed both a higher graded effect size and a higher level of significance in the female study group than in the male study group (see Table 2). In the regression analysis block, the model was significant for both the ODI and RMDQ in the female group, whereas, in the male group, the influence was only significant for the RMDQ. No significant influencing variable was found in the male study group. In the female study group, the DASS score was significant for the ODI and the PHQ-9 
Table 4 Variable Correlation with the Disability Scores ODI and RMDQ According to Patient Sex in the Hierarchical Multivariate Regression Analysis

\begin{tabular}{|c|c|c|c|c|c|c|c|c|}
\hline & \multicolumn{4}{|c|}{ RMDQ } & \multicolumn{4}{|c|}{ ODI } \\
\hline & \multicolumn{2}{|c|}{ Men } & \multicolumn{2}{|c|}{ Women } & \multicolumn{2}{|c|}{ Men } & \multicolumn{2}{|c|}{ Women } \\
\hline & B & $\mathbf{p}$ & B & $\mathbf{p}$ & B & $\mathbf{p}$ & B & $\mathbf{p}$ \\
\hline \multicolumn{9}{|l|}{ Block I } \\
\hline Age & 0.273 & 0.140 & 0.078 & 0.568 & 0.282 & 0.131 & 0.050 & 0.683 \\
\hline BMI & 0.226 & 0.189 & 0.132 & 0.301 & -0.013 & 0.940 & 0.155 & 0.180 \\
\hline Grade of spinal stenosis & 0.100 & 0.560 & 0.206 & 0.116 & -0.008 & 0.962 & 0.206 & 0.081 \\
\hline Pain chronicity & 0.175 & 0.322 & 0.425 & 0.001 & 0.193 & 0.280 & 0.574 & 0.000 \\
\hline \multicolumn{9}{|l|}{ Block 2} \\
\hline Age & 0.302 & 0.075 & 0.085 & 0.501 & 0.300 & 0.106 & 0.126 & 0.900 \\
\hline BMI & 0.188 & 0.231 & 0.053 & $0.64 I$ & -0.035 & 0.837 & 0.052 & 0.606 \\
\hline Grade of spinal stenosis & 0.063 & 0.684 & 0.159 & 0.150 & -0.030 & 0.860 & 0.174 & 0.077 \\
\hline Pain chronicity & 0.086 & 0.598 & 0.347 & 0.001 & 0.138 & 0.442 & 0.510 & 0.000 \\
\hline DASS & 0.428 & 0.058 & 0.216 & 0.147 & 0.276 & 0.257 & 0.302 & 0.022 \\
\hline PHQ-9 & 0.016 & 0.943 & 0.352 & 0.017 & -0.012 & 0.960 & 0.221 & 0.088 \\
\hline \multicolumn{9}{|l|}{ Block 3} \\
\hline Age & 0.232 & 0.177 & 0.050 & 0.685 & 0.201 & 0.296 & -0.032 & 0.770 \\
\hline BMI & 0.130 & 0.403 & 0.011 & 0.930 & -0.064 & 0.712 & 0.029 & 0.786 \\
\hline Grade of spinal stenosis & -0.029 & 0.865 & 0.064 & 0.559 & -0.084 & 0.662 & 0.092 & 0.342 \\
\hline Pain chronicity & 0.159 & 0.348 & 0.315 & 0.002 & 0.231 & 0.227 & 0.473 & 0.000 \\
\hline DASS & 0.398 & 0.094 & 0.124 & 0.396 & 0.186 & 0.475 & 0.201 & 0.122 \\
\hline PHQ-9 & 0.054 & 0.813 & 0.266 & 0.057 & 0.101 & 0.694 & 0.163 & 0.180 \\
\hline Level of pain & 0.268 & 0.067 & 0.313 & 0.007 & 0.271 & 0.098 & 0.198 & 0.048 \\
\hline Pain irradiation & -0.061 & 0.705 & -0.085 & 0.403 & -0.034 & $0.85 I$ & -0.064 & 0.470 \\
\hline Claudication & 0.300 & 0.086 & 0.010 & 0.924 & 0.183 & 0.344 & -0.058 & 0.545 \\
\hline Hyposensation & 0.038 & 0.803 & 0.066 & 0.531 & -0.179 & 0.297 & 0.094 & 0.314 \\
\hline Paresis & 0.124 & 0.439 & 0.113 & 0.290 & 0.167 & 0.357 & 0.198 & 0.039 \\
\hline
\end{tabular}

score for the RMDQ. Therefore, psychological factors have a strong influence on disability scores and a partially altering effect, respectively, to patient sex (see Figure 2).

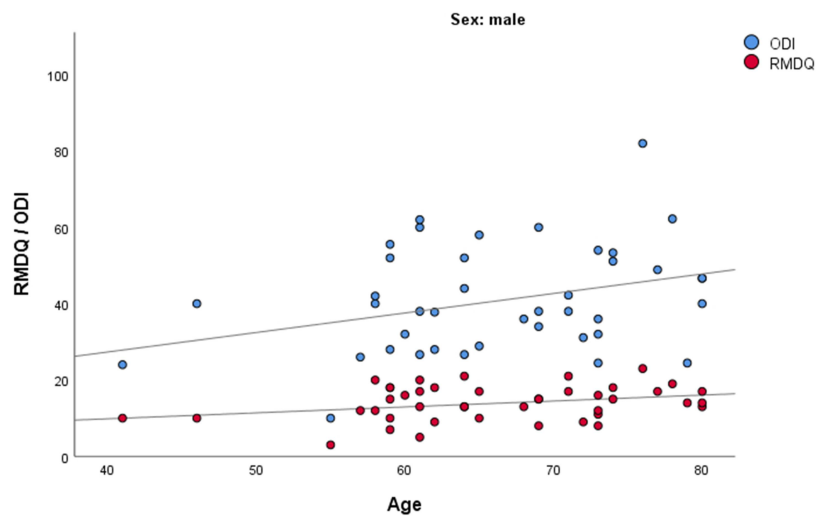

Figure I Correlation of age with the RMDQ and ODI according to patient sex.

\section{Third Block: Symptoms}

Symptom severity was almost equally distributed, without any significant difference, between the two study groups. After adjustment for the baseline variables age and pain

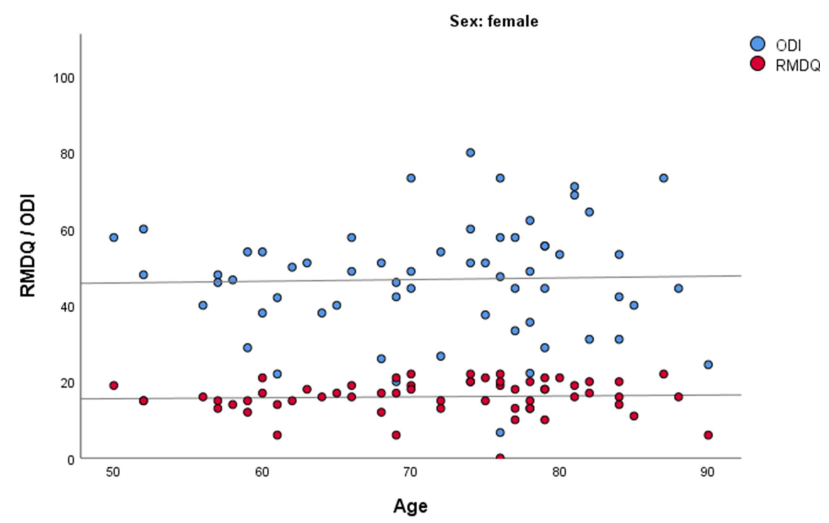



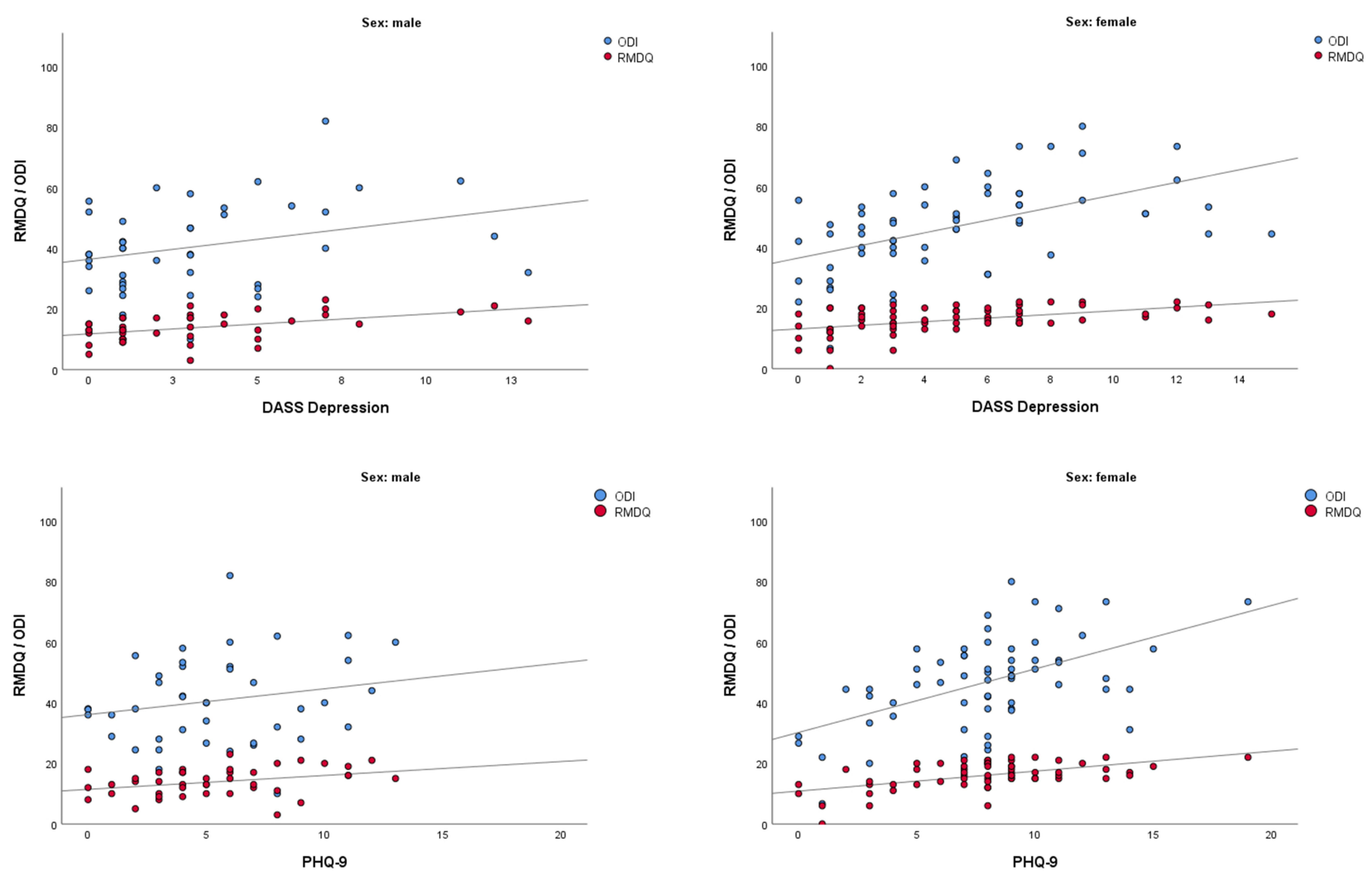

Figure 2 Correlation of DASS and PHQ-9 scores with the RMDQ and ODI according to patient sex.

chronicity, there was no significant difference in level of pain between the study groups $(p=0.885)$. Initially, the level of pain was only significantly correlated with disability scores in the female study group. After adjustment, the male study group showed a significant correlation of the level of pain with ODI and RMDQ, whereas the female study group showed a decrease in the effect size. This finding indicates an influence on disability, but the effect differs between men and women because of cofactors.

The level of pain irradiation was only significantly correlated with ODI and RMDQ in the female study group. Leg pain showed lower ODI and RMDQ scores than isolated back pain. After cofactor adjustment, no significant correlation was observed in either study group. Therefore, pain irradiation only had a cofactordependent influence in the female study group.

Since the severity of claudication symptoms was not significantly correlated with disability scores, it had no significant impact on the findings of this study.

Hyposensation was initially only significantly correlated with the ODI and RMDQ in the female study group. After adjustment, a significant correlation was detected only with the ODI. No significant correlation was found in the male study group. These findings suggest an intermediate influence of hyposensation with cofactordependency in the female study group.

Paretic symptoms only significantly correlated with the ODI and RMDQ in the female study population, indicating an intermediate effect on disability for female patients only.

In the last block of the regression model, the female study group showed a strong influence of somatic variables in both the ODI and RMDQ, but the male study group only showed significant variable dependency in the RMDQ. Focusing on explicit variables within the model, there was no significant regression in the male study group. For the female study group, the level of pain had a significant influence on disability in the ODI and the RMDQ, whereas paresis was an influencing factor in the ODI only.

\section{Discussion}

Next to symptom severity, disability in daily life is one of the key elements in the therapeutic decision-making process. In this study, female patients showed increased impairment in daily life compared to male patients, and 
this finding is coherent with previous studies. ${ }^{16,17}$ The aim of this study is to evaluate possible cofactors for this discrepancy.

Of the baseline data, patient age, pain chronicity, and psychological aspects seemed to be cofactors depending on patient sex. Surprisingly, the male study group but not the female study group showed a significant correlation of age with disability scores, despite the significantly lower mean age of the male study group. This finding suggests that the increased impairment in the female study group cannot solely be attributed to the degenerative physiological processes of advanced age.

In this study, the BMI was not significantly correlated with disability regardless of the sex of the patient. This result is in contrast to other studies predicting higher levels of disability with higher BMI in patients with chronic back pain. ${ }^{18,19}$

As expected, the level of disability was not associated with the radiological grading of the stenosis regardless of patient sex. This finding supports the results of previous studies concluding that the clinical impression of spinal stenosis is independent of the anatomic disposition. ${ }^{20-22}$

The authors consider illness duration a multifactorial process that is best displayed by means of the four axes of the MPSS. The results of this study confirm earlier findings implying a higher multifactorial (social, quality of life, and health-economic) burden for female patients caused by the illness. ${ }^{23,24}$ Longer illness duration correlates with more rigid disability and negatively affects therapeutic outcome. ${ }^{25,26}$ The correlation of the variables in the hierarchical multivariate regression model also supports the assumption that the decrease in Quality of life is influenced by the duration of the illness in female patients predominantly.

Psychological mechanisms such as pain catastrophizing may influence subjective symptom severity as well as therapeutic outcome. ${ }^{27-29}$ The results of this study support earlier findings that female patients with chronic pain tend to be more affected psychologically than male patients, ${ }^{16,30}$ whereas another study states the opposite. ${ }^{31}$ Psychological affection may act as a mediator between symptom severity and subjective disability in daily life. ${ }^{32,33}$

Regarding symptoms, sex-dependent factors include the level of pain and paretic symptoms. Hyposensation and leg pain seem to be influencing factors with a prominent cofactor dependency according to patient sex.

Interestingly, the level of pain was equally distributed between the male and female study populations, whereas, congruent to earlier findings, disability was rated higher for women. ${ }^{34}$ The dynamics of the correlations in this study suggest an intensifying influence of the cofactors on pain-related disability in the female study group and the opposite effect in the male study group. This suggestion is coherent with a meta-study attesting that the factors that influence pain perception differ between men and women. ${ }^{35}$

With regard to pain irradiation, the sole presence of back pain seemed to be a factor influencing disability in the female study group, which was coherent with the findings of a previous study. ${ }^{36}$ This constellation may be due to the higher density of sensory nerve fibers in the Flava ligaments of women compared to men. ${ }^{37}$

In general, reduced walking distance is considered one of the main symptoms of LSS. ${ }^{38}$ The non-significant correlation of claudication with disability scores was surprising as it is an essential element of the scores, but this result may have been caused by inappropriate scaling. However, it may also express the irrelevance of the walking distance for the severity of the LSS regarding the quality of life. Nevertheless, would it be inappropriate to rule out the measurement of the walking distance based on findings of a single study, but it is noteworthy that physical impairment and quality of life regarding this aspect may not be assessed with the same value. Further studies are indicated to validate the results of this study.

Hyposensation correlations indicated an intermediate influencing effect in female patients only. As a previous study has identified a higher vibration threshold as a cofactor for disability variance, ${ }^{39}$ further studies are required to evaluate its relevance.

Regarding the correlation of paretic symptoms with disability, it has been suggested that women give more weight to physical impairment when analyzing daily activity. The overall high correlation in this study validated the importance of paresis symptoms for disability. ${ }^{39}$

Furthermore, the dynamics of the correlations within the female study group before and after adjustment for cofactors implicate an intensifying influence of the psychological status on somatic symptoms. As for the male study group, this effect is reversed for the perception of the level of pain. The correlation of the psychological scores within the second block of the regression model supports this thesis.

As a summary of the findings of this study, the authors suggest that female and male patients with LSS have a different approach to disability in their daily life. Female patients tend to more frequently develop depressive 
disorders than men with the same symptom severity. In accordance with previous studies, the main mediator between symptom severity and disability may be the different pain perception and a higher psychosomatic influence. Thus, the authors advocate to evaluate the factors for disability of patients with LSS according to patient sex.

\section{Limitations}

The study was conducted as a single-center study; therefore, local predispositions in the general population may limit the validity of the study results for the entire cohort of patients with LSS. No matched-pair analysis was applied in this study that would have described the differences between the two sexes to a greater extent.

\section{Abbreviations}

LSS, lumbar spinal stenosis; R, correlation coefficient; $\mathrm{P}$, p-value; cR2, corrected squared regression coefficient; B, standardized coefficient; SSHQ, self-administered selfreported health questionnaire; CSF, cerebrospinal fluid; ODI, Oswestry Disability Index; RMDQ, Roland Morris Disability Questionnaire.

\section{Disclosure}

The authors report no conflicts of interest in this work.

\section{References}

1. Kim YU, Kong Y-G, Lee J, et al. Clinical symptoms of lumbar spinal stenosis associated with morphological parameters on magnetic resonance images. Eur Spine J. 2015;24(10):2236-2243. doi:10.1007/ s00586-015-4197-2

2. Benditz A, Grifka J, Matussek J. Lumbar spinal stenosis. From diagnosis to correct therapy. Zeitschrift für Rheumatologie. 2015;74 (3):215-24; quiz 225. doi:10.1007/s00393-014-1500-2

3. Bartley EJ, Fillingim RB. Sex differences in pain: a brief review of clinical and experimental findings. Br J Anaesth. 2013;111(1):52-58. doi:10.1093/bja/aet127

4. Goesling J, Clauw DJ, Hassett AL. Pain and depression: an integrative review of neurobiological and psychological factors. Curr Psychiatry Rep. 2013;15(12):421. doi:10.1007/s11920-013-0421-0

5. Burke NN, Finn DP, Roche M. Neuroinflammatory mechanisms linking pain and depression. Mod Trends Pharmacopsychiatr. 2015;30:3650 .

6. Udeh BL, Costandi S, Dalton JE, Ghosh R, Yousef H, Mekhail N. The 2-year cost-effectiveness of 3 options to treat lumbar spinal stenosis patients. Pain Pract. 2015;15(2):107-116. doi:10.1111/papr.12160

7. Benditz A, Loher M, Boluki D, et al. Positive medium-term influence of multimodal pain management on socioeconomic factors and health care utilization in patients with lumbar radiculopathy: a prospective study. J Pain Res. 2017;10:389-395. doi:10.2147/JPR.S128090

8. Gilbody S, Richards D, Brealey S, Hewitt C. Screening for depression in medical settings with the Patient Health Questionnaire (PHQ): a diagnostic meta-analysis. J Gen Intern Med. 2007;22(11):1596-1602. doi:10.1007/s11606-007-0333-y
9. Lovibond SH, Lovibond PF. Manual for the Depression Anxiety Stress Scales. 2nd. (Psychology Foundation monograph). Sydney, N.S.W.: Psychology Foundation of Australia; 1995.

10. Fairbank JC, Couper J, Davies JB, O'Brien JP. The Oswestry low back pain disability questionnaire. Physiotherapy. 1980;66(8):271273.

11. Roland M, Morris R. A study of the natural history of back pain. Part I: development of a reliable and sensitive measure of disability in low-back pain. Spine. 1983;8(2):141-144. doi:10.1097/00007632198303000-00004

12. Gerbershagen HU. Das Mainzer Stadiensystem der Schmerzchronifizierung: Testanweisung = Mainz pain staging system . Neuss: Janssen-Cilag; ca; 2002.

13. Janda V. Muscle Function Testing. Kent: Elsevier Science; 2014.

14. Schizas C, Theumann N, Burn A, et al. Qualitative grading of severity of lumbar spinal stenosis based on the morphology of the dural sac on magnetic resonance images. Spine. 2010;35(21):19191924. doi:10.1097/BRS.0b013e3181d359bd

15. Cohen J. Statistical power analysis for the behavioral sciences. Hoboken: Taylor and Francis; 2013. Available from: http://gbv. eblib.com/patron/FullRecord.aspx? $\mathrm{p}=1192162$. Accessed March 5, 2021.

16. Kim H-J, Suh B-G, Lee D-B, et al. Gender difference of symptom severity in lumbar spinal stenosis: role of pain sensitivity. Pain Physician. 2013;16(6):E715-E723.

17. Rundell SD, Sherman KJ, Heagerty PJ, et al. Predictors of persistent disability and back pain in older adults with a new episode of care for back pain. Pain Med. 2017;18(6):1049-1062. doi:10.1093/pm/ pnw236

18. Häuser W, Schmutzer G, Brähler E, Schiltenwolf M, Hilbert A. The impact of body weight and depression on low back pain in a representative population sample. Pain Med. 2014;15(8):1316-1327. doi:10.1111/pme. 12458

19. Segar AH, Urban JPG, Fairbank JCT, Judge A. The association between Body Mass Index (BMI) and back or leg pain in patients with spinal conditions: results from the Genodisc Study. Spine. 2016;41(20):E1237-E1243. doi:10.1097/BRS.0000000000001606

20. Burgstaller JM, Schüffler PJ, Buhmann JM, et al. Is there an association between pain and magnetic resonance imaging parameters in patients with lumbar spinal stenosis? Spine. 2016;41(17):E1053E1062. doi:10.1097/BRS.0000000000001544

21. Weber C, Giannadakis C, Rao V, et al. Is there an association between radiological severity of lumbar spinal stenosis and disability, pain, or surgical outcome?: A Multicenter Observational Study. Spine. 2016;41(2):E78-E83. doi:10.1097/BRS.0000000000001166

22. Hong JH, Lee MY, Jung SW, Lee SY. Does spinal stenosis correlate with MRI findings and pain, psychologic factor and quality of life? Korean J Anesthesiol. 2015;68(5):481-487.doi:10.4097/ kjae.2015.68.5.481

23. Davison MA, Vuong VD, Lilly DT, et al. Gender differences in use of prolonged nonoperative therapies before index lumbar surgery. World Neurosurg. 2018;120:e580-e592. doi:10.1016/j. wneu.2018.08.131

24. Adogwa O, Davison MA, Vuong V, et al. Sex differences in opioid use in patients with symptomatic lumbar stenosis or spondylolisthesis undergoing lumbar decompression and fusion. Spine. 2019;44(13): E800-E807. doi:10.1097/BRS.0000000000002965

25. Cushnie D, Thomas K, Jacobs WB, et al. Effect of preoperative symptom duration on outcome in lumbar spinal stenosis: a Canadian Spine Outcomes and Research Network registry study. Spine J. 2019;19(9):1470-1477. doi:10.1016/j.spinee.2019.05.008

26. Ng LCL, Tafazal S, Sell P. The effect of duration of symptoms on standard outcome measures in the surgical treatment of spinal stenosis. Eur Spine J. 2007;16(2):199-206. doi:10.1007/s00586006-0078-z 
27. Probst T, Neumeier S, Altmeppen J, Angerer M, Loew T, Pieh C. Depressed mood differentially mediates the relationship between pain intensity and pain disability depending on pain duration: a moderated mediation analysis in chronic pain patients. Pain Res Manag. 2016;2016:3204914. doi:10.1155/2016/3204914

28. Sinikallio S, Airaksinen O, Aalto T, Lehto SM, Kroger H, Viinamaki H. Coexistence of pain and depression predicts poor 2-year surgery outcome among lumbar spinal stenosis patients. Nord J Psychiatry. 2010;64(6):391-396. doi:10.3109/08039481003759193

29. McKillop AB, Carroll LJ, Battié MC. Depression as a prognostic factor of lumbar spinal stenosis: a systematic review. Spine J. 2014;14(5):837-846. doi:10.1016/j.spinee.2013.09.052

30. Bingefors K, Isacson D. Epidemiology, co-morbidity, and impact on health-related quality of life of self-reported headache and musculoskeletal pain - a gender perspective. Eur J Pain. 2004;8(5):435-450. doi:10.1016/j.ejpain.2004.01.005

31. Burri A, Rice D, Kluger N, Kluger M. Ethnic- and sex-related differences in pain characteristics, psychological distress and painrelated disability in patients attending a New Zealand teaching hospital pain service. NZ Med J. 2018;131(1470):51-64.

32. Sinikallio S, Aalto T, Airaksinen O, et al. Depression and associated factors in patients with lumbar spinal stenosis. Disabil Rehabil. 2006;28(7):415-422. doi:10.1080/09638280500192462

33. Dysvik E, Natvig GK, Eikeland O-J, Lindstrøm TC. Coping with chronic pain. Int J Nurs Stud. 2005;42(3):297-305. doi:10.1016/j. ijnurstu.2004.06.009
34. Patel DV, Yoo JS, Karmarkar SS, Lamoutte EH, Singh K. Sex differences on postoperative pain and disability following minimally invasive lumbar discectomy. Clin Spine Surg. 2019;32(10):E444E448.doi:10.1097/BSD.0000000000000848

35. Pieretti S, Di Giannuario A, Di Giovannandrea R, et al. Gender differences in pain and its relief. Ann Ist Super Sanita. 2016;52 (2):184-189. doi:10.4415/ANN_16_02_09

36. Alodaibi FA, Fritz JM, Thackeray A, Koppenhaver SL, Hebert JJ. The Fear Avoidance Model predicts short-term pain and disability following lumbar disc surgery. PLoS One. 2018;13(3):e0193566. doi:10.1371/journal.pone.0193566

37. Benditz A, Sprenger S, Rauch L, Weber M, Grifka J, Straub RH. Increased pain and sensory hyperinnervation of the ligamentum flavum in patients with lumbar spinal stenosis. J Orthop Res. 2019;37 (3):737-743. doi:10.1002/jor.24251

38. Tomkins-Lane C, Melloh M, Lurie J, et al. Consensus on the clinical diagnosis of lumbar spinal stenosis: results of an International Delphi Study. Spine. 2016;41(15):1239-1246. doi:10.1097/ BRS.0000000000001476

39. Lin S-I, Lin R-M, Huang L-W. Disability in patients with degenerative lumbar spinal stenosis. Arch Phys Med Rehabil. 2006;87 (9):1250-1256. doi:10.1016/j.apmr.2006.05.021

\section{Publish your work in this journal}

The Journal of Pain Research is an international, peer reviewed, open access, online journal that welcomes laboratory and clinical findings in the fields of pain research and the prevention and management of pain. Original research, reviews, symposium reports, hypothesis formation and commentaries are all considered for publication. The manuscript management system is completely online and includes a very quick and fair peer-review system, which is all easy to use. Visit http:// www.dovepress.com/testimonials.php to read real quotes from published authors. 